\title{
Théologiques
}

\section{Autres regards sur la laïcité}

\section{Solange Lefebvre}

Volume 6, numéro 1, mars 1998

Autres regards sur la laïcité

URI : https://id.erudit.org/iderudit/016654ar

DOI : https://doi.org/10.7202/016654ar

Aller au sommaire du numéro

Éditeur(s)

Faculté de théologie de l'Université de Montréal

ISSN

1188-7109 (imprimé)

1492-1413 (numérique)

Découvrir la revue

Citer ce document

Lefebvre, S. (1998). Autres regards sur la laïcité. Théologiques, 6(1), 3-7.

https://doi.org/10.7202/016654ar d'utilisation que vous pouvez consulter en ligne.

https://apropos.erudit.org/fr/usagers/politique-dutilisation/ 
LIMINAIRE

\title{
Autres regards sur la laïcité
}

\author{
La sécularisation des mœurs, le pluralisme, la séparation \\ de l'Église et de l'État, tous ces thèmes fertiles en idées reçues ne \\ sont en fait qu'un humus récent et inégalement réparti, reposant sur une \\ histoire longue avec laquelle nous n'avons pas fini de compter'.
}

Le terme «laïcité " semble se trouver très marqué par la langue et le contexte, tellement qu'il se trouve apparemment captif d'un usage somme toute restreint, voire même national. Il fit son apparition en France, en 1871, et s'imposa peu à peu dans les Dictionnaires. Les mots « laïciser " et "laïcisation" apparurent au début de la III e République. Les termes laïcité et ses dérivés réfèrent en ce sens à une réalité politique très spécifique, qui se trouve au-delà de l'idée de neutralité qui s'y rattache souvent : un "radical affranchissement à l'égard de toute autorité transcendante à la raison ${ }^{2}$ ". Condorcet ne partageait-il pas le genre humain en deux classes? Ceux qui raisonnent et ceux qui croient, les maîtres et les esclaves...? Selon le collectif de Montpellier, "il fallait donc un mot nouveau - qui reste aujourd'hui encore intraduisible en allemand ou en anglais » (BOST 1990: 7).

Pourtant, réfléchir sur la laïcité dans une perspective multidisciplinaire, signifie s'aventurer sur des terrains fortement sédimentés. Catholiques, protestants, hérétiques, laïcistes, systèmes politiques, intellectuels et gens du peuple y ont laissé quantité de sédiments successifs. Elle se trouve, pour reprendre les termes de Michel Foucault, "prise dans l'épaisseur d'un cumul (qu'elle) ne cesse pourtant de modifier, d'inquiéter, de bouleverser et parfois de ruiner $(1969: 164)$. C'est ce qu'illustre la diversité des points de vue présentés dans ce numéro de Théologiques. On pourrait résumer ainsi cette tentative : la laïcité ne saurait s'expliquer

1 Jean-Pierre MANIGNE. 1988. "Laïcité: la fin des naïvetés ", L'Actualité religieuse dans le monde (15 mars 1988) 36.

2 Louis CAPÉRAN, Histoire contemporaine de la laïcité française. T. 1, Paris, 1957, p. XXV; cité dans BOST 1990, p. 8. 
uniquement par les décrets des institutions et du pouvoir, mais aussi par la culture et de l'histoire chrétiennes et occidentales qui la portent.

Au départ du projet de ce numéro, nous rêvions d'un "autre regard » sur la laïcité. Celle-ci demeurait une problématique surtout européenne, mais pourtant le mot surgissait ça et là dans l'actualité, dans certains écrits, sans trop d'explicitation. Nos auteurs, tous québécois, ont le mérite de commencer à mettre au jour l'histoire commune qui nous lie aux pays initiateurs de la laïcité (au sens large et non strictement français), et d'en réfléchir la portée chez nous, à la fois autour et pourtant bien au-delà de la question révélatrice de la "religion à l'école».

La sociologue de la religion, Micheline Milot, veut montrer en quoi l'idée de laïcité déborde le contexte idéologique français anticlérical dont elle est issue : il s'agit d'une réalité évolutive. D'abord, la laïcité fait l'objet d'interprétations diverses, et surtout, elle "n'exprime pas une opinion sur la religion, mais une opinion sur l'État ... À l'origine, c'est essentiellement une conception de la neutralité de l'État et de l'enseignement qui sert à définir la laïcité». Suivront la séparation entre les religions et l'État, sur fond de luttes entre l'État laïque et l'Église qui se disputent le monopole du pouvoir. Mais depuis, la laïcité a exercé sur elle-même une critique, si bien qu'à présent, elle se résume en l'aménagement d'un espace public où sont garanties la liberté religieuse et l'égalité des individus.

Milot relève aussi une autre distinction importante entre l'État, la sphère privée et la société civile. Et les religions peuvent continuer de jouer un rôle "social " au sein de cette dernière. À ce titre, la séparation entre l'Église et l'État a permis le développement, au sein de la société civile, d'une « religiosité moins régulée, plus individualiste et délibérative». Mais de nouvelles questions se posent à l'État, qui ne peut demeurer dans une pure neutralité à l'égard des effets de la diversité religieuse sur le lien social. Dans les faits en outre, malgré la reconnaissance du pluralisme religieux, les religions dominantes conservent certains privilèges. Milot examine la question scolaire, comme lieu révélateur des enjeux de la laïcité présentement.

L'anthropologue Jacques T. Godbout critique le fondement de la lä̈cité française: la raison abstraite qui rejette tout lien communautaire significatif voulant se développer en son sein. Celui-ci serait vu comme un particularisme qui renvoie à l'ordre des privilèges de l'Ancien Régime et qui menace l'État de droit. Cette vision des choses occulte le fondement 
du lien social échappant à la raison : le fondement indisponible de la normativité. Godbout préfère le modèle britannique du common law, qui ramène le pouvoir aux réseaux sociaux, "en "rabaissant" ce complexe, orgueilleux et rationnel appareil politique moderne (...) à cette modeste base qu'est la coutume, la façon dont les ancêtres ont réglé le problème, non pas pour s'y conformer (...) mais pour s'en inspirer et prendre une décision "éclairée" par autre chose que la raison abstraite" .

Pour situer les propos de Godbout, quant aux rapports entre État et religion, ajoutons que le Royaume-Uni présente des similitudes avec l'Allemagne, exception faite de certains avantages fiscaux. Il reconnaît légalement deux Églises d'État, l'anglicane et la presbytérienne. La fonction royale se combine à celle de chef de l'Église anglicane, et la Chambre des Lords comprend parmi ses membres des dignitaires ecclésiastiques, auxquels s'est joint récemment le grand rabbin d'Angleterre. L'éducation religieuse chrétienne est offerte dans les écoles, mais les parents qui le désirent peuvent l'éviter. Les textes constitutionnels ne reconnaissent pas explicitement les libertés de religion, mais celles-ci "découlent légalement et pratiquement de la notion de tolérance " (ZYLBERBERG 1995: 42). La population britannique, comme l'allemande, est très sécularisée dans sa vie quotidienne, mais la référence et l'appartenance religieuses demeurent symboliquement importantes.

L'approche de Godbout s'inscrit dans le courant qui préfère la laïcité de cohabitation, ou coexistence anglaise, à la laïcité française, " idéologique et niveleuse ${ }^{3}$ ». Et la relation entre démocratie et religion renvoie aux liens complexes, dans la modernité, entre démocratie et religion, entre démocratie et nationalisme, entre le politique et le social, entre l'universalisme et l'identité. Plus généralement, la démocratie moderne contractuelle tendrait vers l'éradication du particulier en même temps qu'elle en active la prolifération (THÉRIAULT 1996).

Avec les articles de Pichette, Larose et Lefebvre, la laïcité se voit examinée dans ses fondements et ses origines. Jean Pichette, fait un travail d'histoire des idées et décèle les apories de la laïcité, captive du positivisme hérité du dualisme ontologique chrétien. Les querelles entre clercs et laïcs ont conduit à l'élimination du premier terme, abolissant la nécessité d'un détour par une altérité pour penser la condition humaine autonome. gration, plutôt qu'une laïcité de coexistence communautariste. 
Pichette examine les rapports entre l'éducation laïque et son contexte épistémologique. Le positivisme d'Auguste Comte, repris dans le projet de Jules Ferry, réifie la société. Discutant la thèse de la sécularisation comme transfert d'idées chrétiennes dans la sphère laïque, il fait une autre lecture de la modernité, comme «approfondissement de la césure à travers laquelle le sujet et l'objet peuvent naître au monde, au point de les renvoyer dans deux sphères ontologiques radicalement disjointes ». Quant à l'instance séparatrice, l'altérité, elle se voit reportée dans le sujet rationnel, pourtant incapable de fonder par lui-même la société. Ce qui est appelé, c'est un nouveau lien entre les deux ordres séparés par le christianisme, entre la transcendance et le profane. Le "apprendre à prier " de l'agnostique Schönberg, signifierait se réapproprier un monde abandonné aux puissances technocratiques.

Jean Larose, sous le mode de l'essai, fait quant à lui une lecture psychanalytique et littéraire du rapport trouble à notre matrice chrétienne. Il observe cette particularité des pays d'origine chrétienne: l'hostilité ou l'indifférence modernes à l'égard de sa propre souche religieuse, justement. Pourrait-on attribuer cette hostilité au fait que le christianisme est " une affaire de famille "?

Une lecture de Malraux et de Bossuet fait ressortir du christianisme les traits d'une grande sensibilité et d'une grande humanité. Nulles autres cultures au monde ne font montre d'une telle capacité à la compassion, à la culpabilité et à la vulnérabilité à l'égard d'autrui. Ce que Larose relie à "l'idée compassionnelle de l'amour dont le catholicisme a empreint notre civilisation". Amour et loi forment ce "mélange insupportable " qui établit entre le sujet et Dieu une relation mue par le désir, et qui nourrit en quelque sorte "l'inconscient de la laïcité ». Cet amour est mis à mal par l'hédonisme moderne, et les masses se jettent dans les bras d'Isis, cette Déesse du Nouvel Âge sans interdit et sans dogme.

Solange Lefebvre retrace la mémoire du mot "laïcité ", et les échos qui y retentissent, singulièrement contemporains : plus de cent ans après l'élaboration du projet de laïcité en France, qui rendait la raison souveraine et l'émancipait d'une autorité transcendante, nous nous trouvons renvoyés aux tensions historiques entre clercs et laïcs autour de la domination de la sphère spirituelle. Présentement, le phénomène de désinstitutionnalisation de la religion évoque ces tensions, c'est-à-dire que l'individualisation contemporaine de la croyance origine pour une part de la subjectivisation chrétienne laïque de l'expérience religieuse qui s'est affirmée surtout à partir du Moyen Âge. La référence aux clercs n'a pas pour autant été éliminée, même si elle a muté, ce qui ressort dans les nouveaux rapports des modernes aux traditions, et plus spécifiquement aux institutions religieuses. Cette mutation renvoie à une révision fondamen- 
tale moderne de la dichotomie entre sacré et profane. Même s'il fut forgé pour exprimer tout autre chose au XIX ${ }^{e}$ siècle, le mot "laïcité " revêt une prégnance théologique profonde.

\section{Bibliographie}

BACZKO, Bonilaw. 1992. «L'héritage de la Révolution française», dans Dieux en sociétés. Le religieux et le politique (FUGUIER, Richard, dir.). Paris, Autrement (série "Mutations"127), pp. 73-87.

BOST, Hubert (dir.). 1990. Genèse et enjeux de la laïité. Christianisme et laïcité. Genève, Labor et Fides, 228 p.

FOUCAULT, Michel. 1969. L'archéologie du savoir. Paris, Gallimard, $275 \mathrm{p}$.

HAARSCHER, Guy. 1996. La laïité. Paris, Cerf (Que sais-je? 3129), $123 \mathrm{p}$.

THÉRIAULT, Jacques-Yvon. 1996. "Le démocratisme et le trouble identitaire ", dans Les frontières de l'identité (ELBAZ, Michaël, dir.). SainteFoy/Paris, PUL/L'Harmattan, pp. 165-179.

ZYLBERBERG, Jacques. 1995. "Laïcité, connais pas: Allemagne, Canada, États-Unis, Royaume-Uni » dans Pouvoirs, La laïcité. Paris, Seuil, pp. 37-52. 